\title{
Os Efeitos do Trabalho em Turnos no Controle Metabólico de Pacientes Diabéticos
}

$\mathrm{O}_{\mathrm{r}}$ DiABETES MELITO (DM) tipo 2 é uma doença com elevada e crescente prevalência, incluindo pacientes cada vez mais jovens, o que determina um aumento da doença em uma população de pacientes em faixa etária produtiva. Os custos associados ao tratamento do DM e suas complicações produzem uma elevada carga econômica para os pacientes, familiares e para toda a sociedade. Indivíduos com DM possuem maior risco de incapacidade para o trabalho (1). As causas dessas incapacidades funcionais são diversas, entre elas: as complicações crônicas decorrentes da doença, as co-morbidades associadas, o excesso de peso, o despreparo físico, os sintomas de descompensação aguda e depressão (2). Um estudo que avaliou a prevalência de diabetes em uma grande empresa brasileira demonstrou uma prevalência de $11,5 \%$ entre os trabalhadores dessa empresa, além da ausência de relação do trabalho com a doença. Homens acima dos 40 anos de idade apresentaram um risco maior para o desenvolvimento de diabetes (3).

O trabalho em turnos, especialmente o trabalho noturno, está associado a alguns distúrbios característicos, entre eles: fadiga crônica, alteração do padrão do sono e alteração do funcionamento intestinal. $\mathrm{O}$ trabalho em turnos é um fator de risco para o início de DM, em especial a sua alternância (1).

Indivíduos jovens, com até 50 anos de idade, que trabalham à noite possuem níveis mais elevados de triglicerídeos e de glicemia, assim como níveis mais baixos de HDL colesterol, quando comparados aos indivíduos que trabalham apenas durante o dia (4). Entretanto, o efeito do trabalho noturno no controle glicêmico de pacientes portadores de DM é pouco conhecido.

Realizamos uma avaliação transversal em 100 pacientes com DM tipo 2, todos funcionários do Hospital de Clínicas de Porto Alegre, em diferentes funções. Os pacientes foram atendidos no ambulatório de Medicina Interna do Serviço de Medicina Ocupacional do mesmo hospital no período de janeiro de 2004 a julho de 2006. Destes, 67 trabalhavam no turno diurno e 28 no turno noturno. Cinco pacientes foram excluídos por realizarem alternância de turno. As características clínicas e laboratoriais estão descritas na tabela 1 . A presença de nefropatia diabética foi definida quando os pacientes apresentaram excreção urinária de albumina (EUA) > $30 \mathrm{mg} / 24 \mathrm{~h}$. Para a classificação de síndrome metabólica (SM) foram utilizados os critérios do National Cholesterol Education Program (NCEP).

Não observamos diferenças entre controle metabólico e complicações renais em relação ao turno de trabalho em pacientes com DM tipo 2 e funcionários de um hospital universitário. A diferença significativa encontrada entre os grupos foi maior obesidade abdominal nos pacientes que trabalhavam à noite; além disso, observamos uma tendência a níveis mais elevados de pressão arterial diastólica em trabalhadores noturnos. A literatura traz resultados diversos entre a relação de turnos de trabalho e controle metabólico, mas níveis elevados de pressão arterial, hipertrigliceridemia e hiperglicemia já foram observados em trabalhadores

\section{carta ao editor}

\section{TICIANA C. RODRIGUES \\ luis Henrique S. Canani}

\author{
Serviço de Medicina \\ Ocupacional e Serviço de \\ Endocrinologia, Hospital de \\ Clínicas de Porto Alegre, \\ Universidade Federal do \\ Rio Grande do Sul, \\ Porto Alegre, RS.
}

Recebido em 06/03/07

Revisado em 20/07/07

Aceito em 12/09/07 
Tabela 1. Características clínicas e laboratoriais de pacientes com diabetes melito tipo 2 de acordo com o turno de trabalho.

\begin{tabular}{|c|c|c|c|}
\hline & $\begin{array}{c}\text { Diurno } \\
(n=67)\end{array}$ & $\begin{array}{l}\text { Noturno } \\
(n=28)\end{array}$ & $\mathbf{P}$ \\
\hline Idade (anos) & $46,55 \pm 9,0$ & $48,42 \pm 6,6$ & 0,32 \\
\hline Duração do DM (anos) & $4,44 \pm 4,1$ & $3,45 \pm 3,5$ & 0,32 \\
\hline Sexo Masculino - $n(\%)$ & $18(26,86)$ & $6(21,42)$ & 0,69 \\
\hline Caucasianos - n (\%) & $41(61,20)$ & $16(57,14)$ & 0,93 \\
\hline Hipertensos - n (\%) & $33(49,25)$ & $12(42,85)$ & 0,56 \\
\hline Pressão Arterial Sistólica $(\mathrm{mmHg})$ & $124,38 \pm 13,56$ & $127,60 \pm 12,67$ & 0,29 \\
\hline Pressão Arterial Diastólica (mmHg) & $79,00 \pm 14,12$ & $83,80 \pm 8,32$ & 0,05 \\
\hline IMC $\left(\mathrm{kg} / \mathrm{m}^{2}\right)$ & $30,52 \pm 5,42$ & $32,97 \pm 6,25$ & 0,09 \\
\hline Cintura abdominal $(\mathrm{cm})$ & $95,93 \pm 11,58$ & $103,57 \pm 13,72$ & 0,01 \\
\hline Glicemia de jejum (mg/dl) & $148,90 \pm 60,4$ & $153,2 \pm 58,1$ & 0,75 \\
\hline Hemoglobina $A_{l c}(\%)$ & $7,72 \pm 1,92$ & $7,29 \pm 1,72$ & 0,30 \\
\hline Colesterol total (mg/dl) & $193,00 \pm 35,42$ & $195,32 \pm 35,32$ & 0,78 \\
\hline Colesterol HDL (mg/dl) & $48,11 \pm 11,35$ & $49,52 \pm 9,90$ & 0,58 \\
\hline Triglicerídeos (mg/dl) & $149(107-496)$ & $130(34-302)$ & 0,36 \\
\hline Creatinina Sérica $(\mathrm{mg} / \mathrm{dl})$ & $0,97 \pm 0,34$ & $0,86 \pm 0,14$ & 0,30 \\
\hline Excreção urinária de albumina (mg/24h) & $7,60(0,00-138,80)$ & $29,95(0,00-327,60)$ & 0,15 \\
\hline normo/micro/macroalbuminúria (\%) & $47,8 / 20,8 / 1,5$ & $17,9 / 10,4 / 1,5$ & 0,72 \\
\hline Síndrome Metabólica — n (\%) & $36(54,5)$ & $17(61,5)$ & 0,64 \\
\hline
\end{tabular}

Dados expressos em médias \pm DP, \% ou mediana (variação); DM: diabetes melito; IMC: índice de massa corporal

noturnos com menos de 50 anos de idade (4). No presente estudo, a circunferência da cintura foi diferente entre os grupos. O significado dessa maior obesidade abdominal não é claro, podendo representar maior resistência à insulina (RI) nesse grupo de pacientes. Entretanto, outros marcadores de RI, entre eles triglicerídeos, microalbuminúria e $S M$, não foram diferentes entre os grupos. Apesar de outros fatores da RI não estarem presentes, a obesidade abdominal é um fator de risco independente para mortalidade em pacientes com DM (5).

Em conclusão, nossos dados não sugerem que o turno de trabalho piore o controle metabólico em indivíduos com DM. O aumento da medida da circunferência abdominal nos trabalhadores noturnos pode identificar um grupo em risco para eventos cardiovasculares. É necessária uma investigação prospectiva para a melhor avaliação da relação de controle metabólico e turnos de trabalho, assim como da caracterização da RI.

\section{REFERÊNCIAS}

1. Mayfield JA, Deb P, Whitecotton L. Work disability and diabetes. Diabetes Care 1999;22:1105-9.

2. Valdamis V, Smith DW, Page MR. Productivity and economic burden associated with diabetes. Am J Public Health 2001;91:129-30.

3. Martinez MC, de Oliveira Latorre MRD. Risk factors for hypertension and diabetes mellitus in metallurgic and siderurgic company's workers. Arq Bras Cardiol 2006;87:424-32.

4. Nagaya T, Yoshida $H$, Takahashi $H$, Kawai M. Markers of insulin resistance in day and shift workers aged $30-59$ years. Int Arch Occup Environ Health 2002;75:562-8.

5. Despres JP. Intra-abdominal obesity: an untreated risk factor for type 2 diabetes and cardiovascular disease. J Endocrinol Invest 2006;29:77-82.

\section{Endereço para correspondência:}

Ticiana C. Rodrigues

Serviço de Endocrinologia

Hospital de Clínicas de Porto Alegre

Rua Ramiro Barcelos 2350, prédio 12, $4^{\circ}$ andar

90035-003 Porto Alegre, RS

E-mail: endocrinologista@terra.com. 